\title{
Eine mittelpaläolithische Formengruppe und das Problem ihrer geochronologischen Einordnung ${ }^{1}$ )
}

\author{
Von Gerhard Bosinski, Köln \\ Mit 9 Abbildungen und 1 Tabelle
}

\begin{abstract}
$\mathrm{Z}$ us a m men f a s s u $\mathrm{ng}$ : Levalloisien und Acheuléen sind keine unterschiedlichen Kulturen, sondern lediglich 2 Komponenten eines Komplexes, der, der ursprünglichen Definition folgend, als Acheuléen zu bezeichnen ist. Dem Acheuléen zuzurechnende nordwestdeutsche Funde, zur exakteren Erfassung als Leberstedter Gruppe bezeichnet, werden in ihrem Formenschatz und ihrer geochronologischen Stellung untersucht.
\end{abstract}

A bstract: Levalloisien and Acheuléen are no different cultures but only two components of one complex which is to describe as Acheu'éen, following the original definition. The essay deals with artifacts and geochronological position of some finds in northwestern Germany belonging to the Acheuléen. For these finds I suggest the term Lebenstedter Gruppe.

Ré sumé : Levalloisien et Acheuléen ne sont pas des industries différentes, mais seulement 2 composantes d'un complexe, qui est déclaré comme Acheuléen suivant la définition originale. Les gisements acheuléennes au nord-ouest d'Allemagne déclarées comme Lebenstedter Gruppe sont examinées à leur richesse des formes et à leur position géo-chronologique.

Die folgenden Darlegungen erwuchsen aus meiner Dissertation über „Das Mittelpaläolithikum im westlichen Mitteleuropa“; dort sind die unten angeführten Inventare dokumentiert (Katalog und Tafeln), so daß hier auf weitere Abbildungen verzichtet werden kann.

Bei der Beschreibung und formenkundlichen Charakterisierung der Funde sowie bei der anschließenden Untersuchung zur Chorologie und relativen Chronologie kommen Methoden der Urgeschichte zur Anwendung. Dagegen ist zur geochronologischen Fixierung der hier behandelten mittelpaläolithischen Formengruppe eine Mithilfe der Geologie notwendig.

\section{A. Nordwestdeutsche Fundstellen}

1952 wurden durch die Initiative A. Tode's in der Nähe von Salzgitter-Lebenstedt in einer Tiefe von über $4 \mathrm{~m}$ zahlreiche Artefakte in planmäßiger Ausgrabung geborgen (Vorbericht: Tone 1953) ${ }^{2}$ ). Einige Faustkeile sind vorhanden: Langschmale, spitze Typen mit im Längsschnitt kontinuierlich verdicktem Ende (ToDE 1953, Abb. 7, 1 - S. 201; auch das große Stïck ebenda, Abb. 8 - S. 202 gehört zu diesem Typ); kleinere, annähernd dreieckige Faustkeile, in der Regel nur an den Kanten und an der Spitzenpartie besser bearbeitet; massive, über $10 \mathrm{~cm}$ lange Faustkeile mit verdicktem, nur grob behauenem Ende und im Querschnitt flacher, verrundeter Spitzenpartie (ebda, Abb. 5, 2 - S. 198; Abb. 6, 2 - S. 199). Eine weitere wichtige Werkzeugform sind die in Lebenstedt zahlreichen blattförmigen Schaber: annähernd ovale Artefakte mit bogenförmiger, dorsal und ventral geschärfter Arbeitskante; die Gegenkante ist in sich alternierend behauen, zickzackförmig und als Arbeitskante völlig ungeeignet (ebda, Abb. 14 - S. 209).

Häufig sind einfache Schaber mit leicht konvexer oder annähernd gerader, nur dorsal retuschierter Arbeitskante parallel zur Schlagrichtung (ebda, Abb. 11 - S. 205; Abb. 12 S. 206). Diese Werkzeuge besitzen oft eine fazettierte Schlagfläche, bzw. es handelt sich um L.evalloisabschläge, deren eine Längskante dorsal retuschiert ist. Breitschaber (racloirs

1) Vortrag auf der Deuqua-Tagung 1962 in Nürnberg.

2) Herrn Dr. A. Tode, Braunschweig, danke ich für die Erlaubnis zum ausführlichen Studium der Funde noch vor dem Erscheinen der Lebenstedt-Monographie, die für die Schriftenreihe ,Fundamenta" vorbereitet wird. 

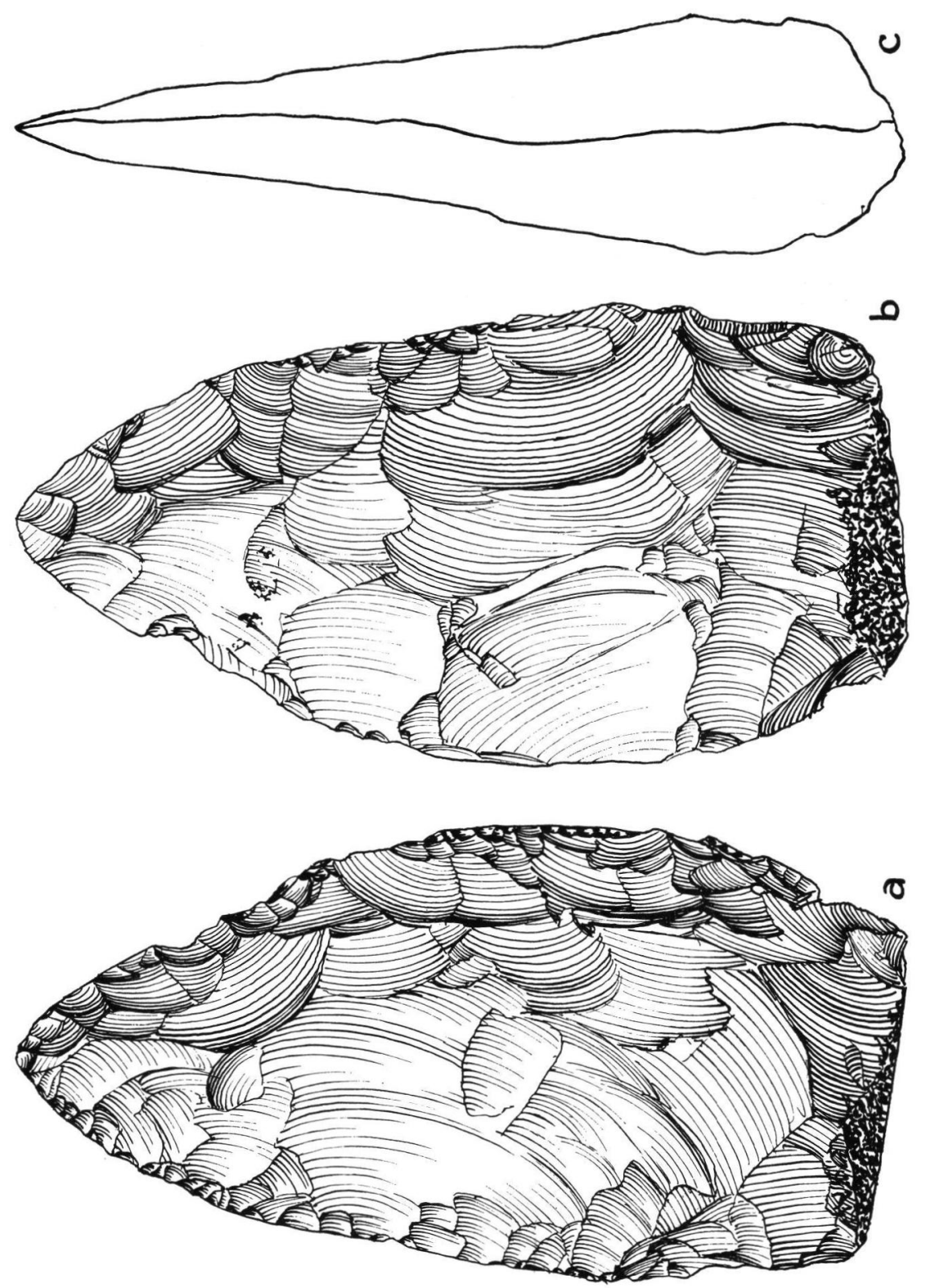

transversaux) fehlen vollständig (die unübliche Orientierung der Schaber ebda, Abb. 11 und Abb. 12, 1-2 könnte zu Verwechslungen Anlaß geben). Schaber mit zwei Arbeitskanten (Doppelschaber, Winkelschaber, Spitzschaber) wurden in Lebenstedt nicht gefunden.

Wichtigste Spitzenform sind große Levalloisspitzen (ebda, Abb. 10, 4 - S. 204). Daneben kommt eine Sonderform vor: breitflache Abschläge, meist mit fazettierter Schlagfläche, deren leicht verrundete Spitzenpartie dorsal im oberen Drittel flächenretuschiert ist (wie das aus Herne abgebildete Stïck: Abb. 4). Weitere Spitzenformen, insbesondere 

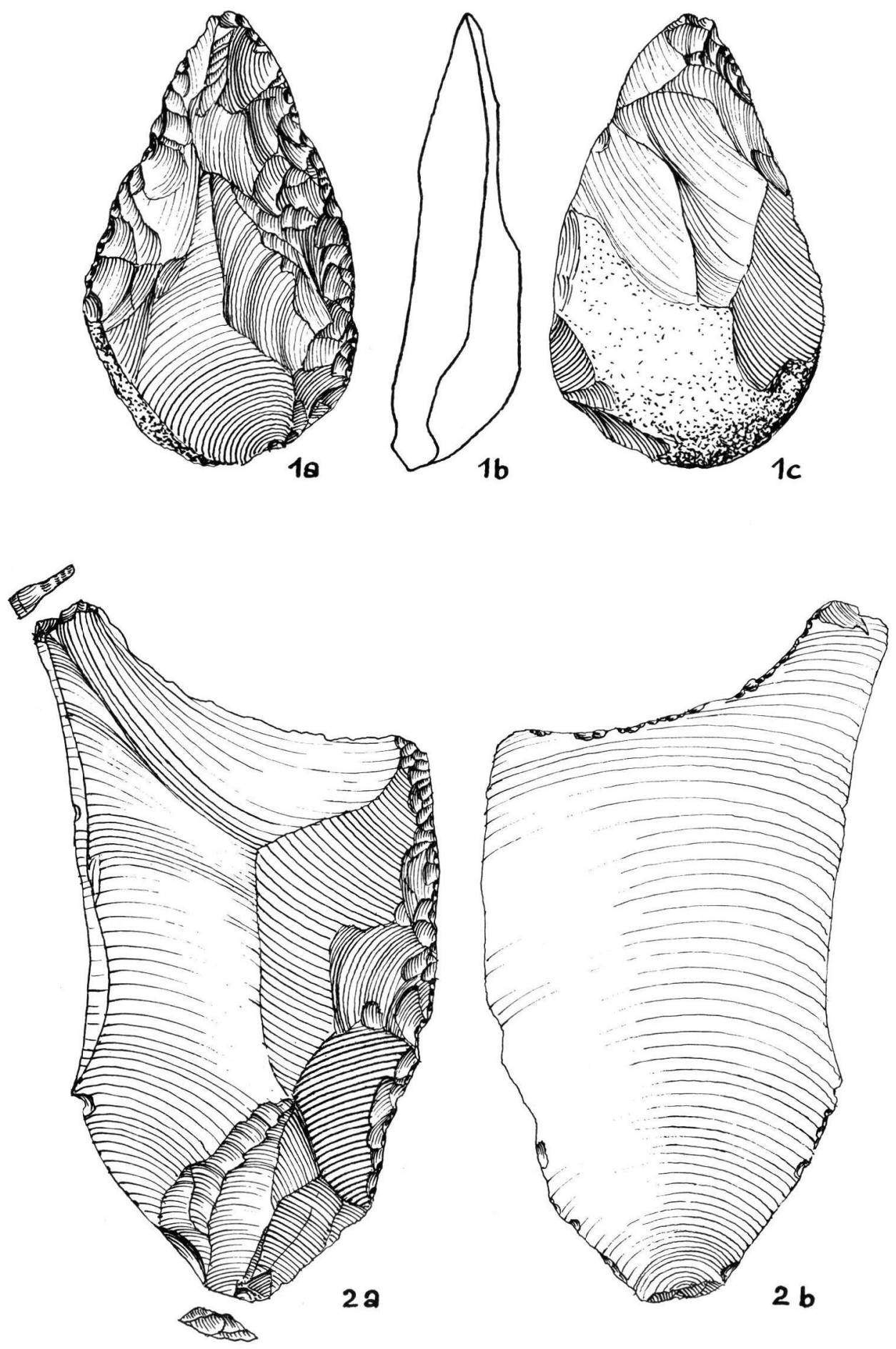

Abb. 2. Herne (1:1). Faustkeil (1) und Schaber (2). 

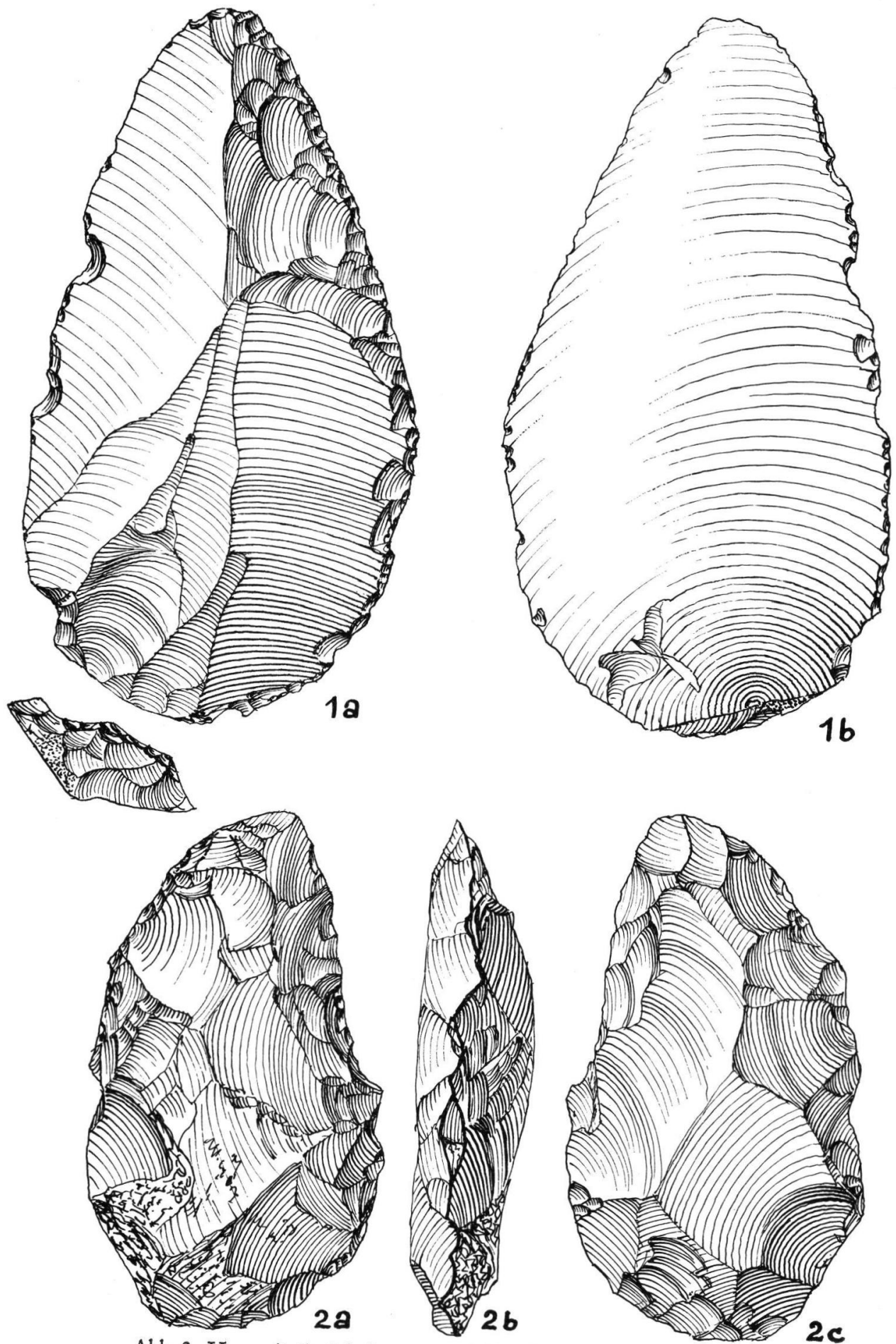

Abb. 3. Herne (1:1). Schaber (1) und blattförmiger Schaber (2)

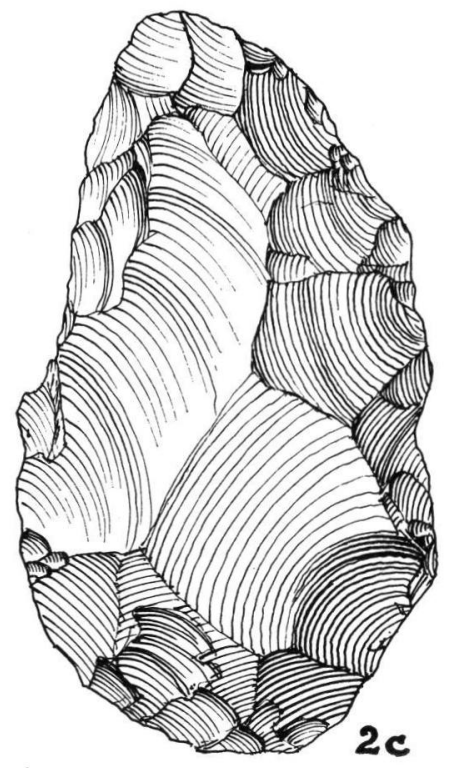



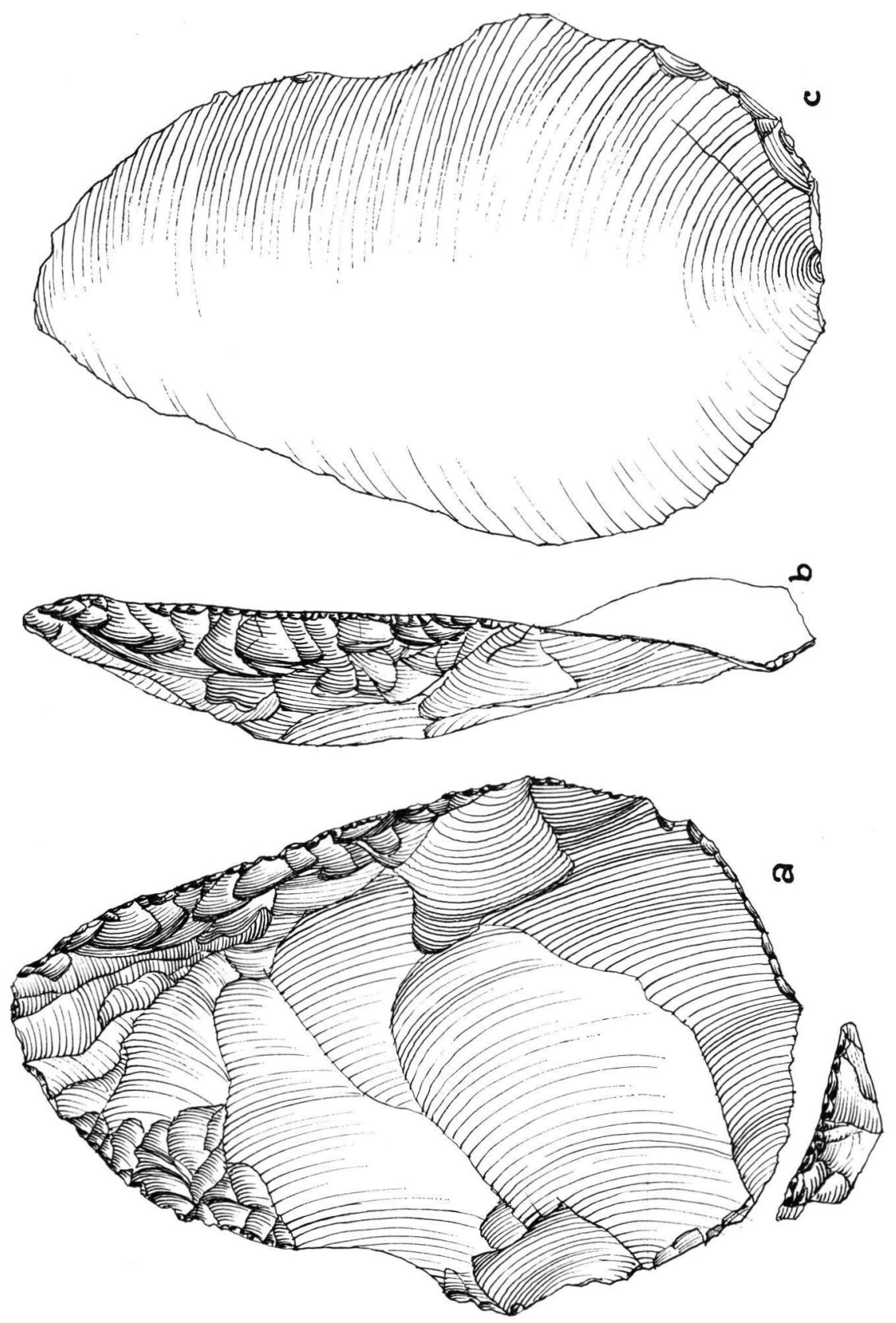

Spitzen mit retuschierten Kanten, fehlen. Schmalklingen, darunter an einer oder beiden Längskanten dorsal retuschierte Stücke, kommen vor. Mitbestimmend für den Charakter des Inventars sind zahlreiche Abschläge von klassifikatorischer Bedeutung: Breitflache, größere Levalloisabschläge (38 Stück; z. B. TodE 1953, Abb. 10 - S. 204) kommen neben kurzdicken Clactonabschlägen (ebda, Abb. 9 - S. 203) vor. Wichtigste Kernsteinform sind 


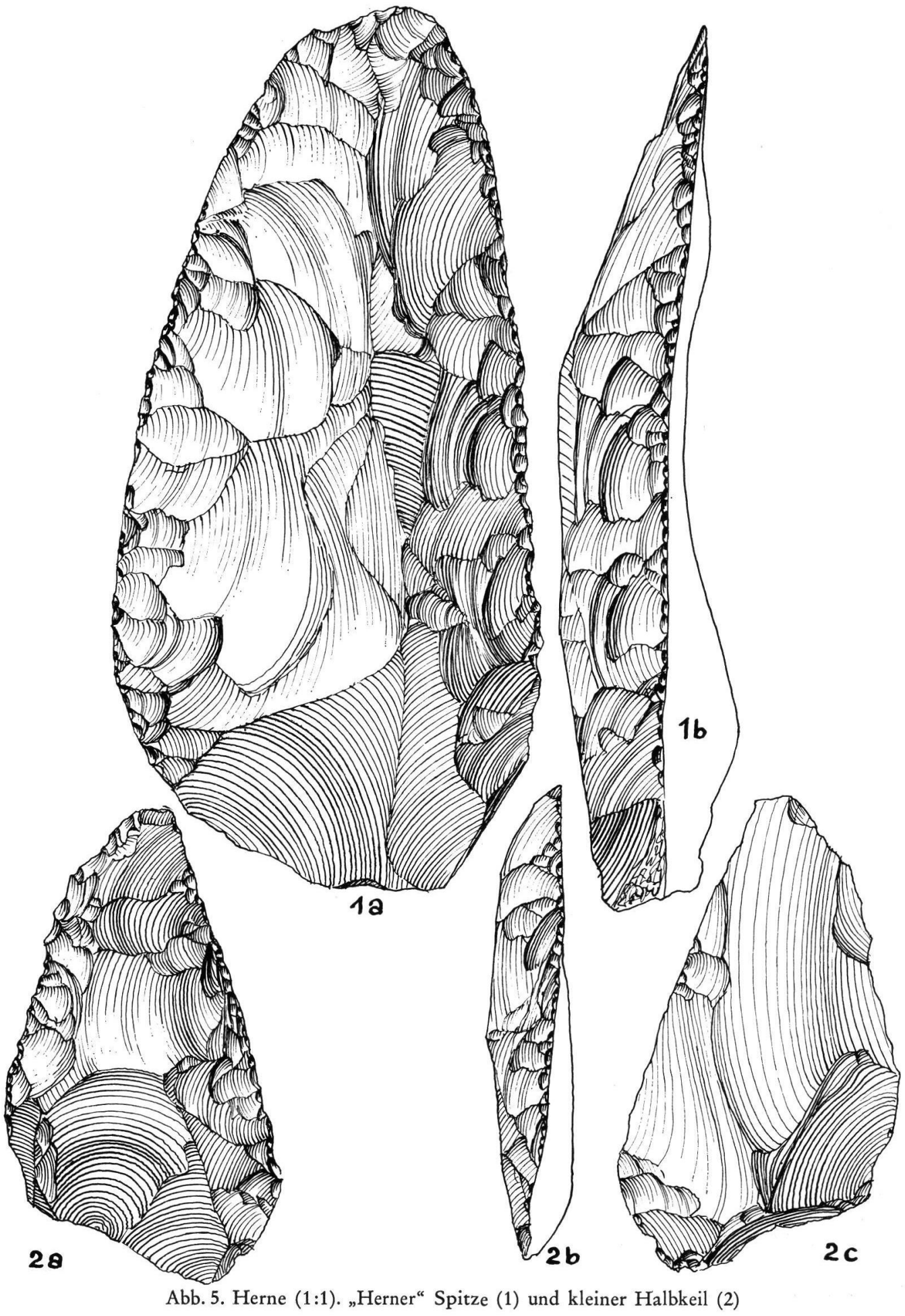

9 Eiszeit und Gegenwart 
Schildkerne; weiterhin bemerkenswert ist ein konischer Nukleus mit glatter Standfläche (wie Abb. 8, 1).

In He r n e konnte E. Kahrs $(1925,1928)$ schon 1911 beim Bau des Rhein-HerneKanals ähnliche Funde bergen. Auch hier sind zunächst Faustkeilformen zu nennen: ein langschmales, spitzes Stück mit gleichmäßig verdicktem Ende (Abb. 1) ${ }^{3}$ ) steht neben kleinen, annähernd dreieckigen Faustkeilen, die, wie in Lebenstedt, nicht völlig durchgearbeitet und nur an den Kanten und an der Spitze besser retuschiert sind (Abb. 2, 1). Die blattförmigen Schaber sind durch ein Stück belegt (Abb. 3, 2 - aufgeklappt ist die der Arbeitskante gegenüberliegende, zickzackförmig verlaufende Kante). Bei den einfachen Schabern (Abb. 2, 2; Abb. 3, 1) handelt es sich gleichzeitig um typische, breitflache Levalloisabschläge, deren ein€ Längskante dorsal retuschiert ist.

Auf die in Abb. 4 wiedergegebene Spitzenform wurde schon in Lebenstedt hingewiesen: ein breiter Abschlag mit leicht verrundetem, nur dorsal im oberen Drittel flächig retuschierten Spitzenteil (Herner Spitze). Auch das Werkzeug Abb. 5, 1, in der Literatur manchmal als Halbkeil bezeichnet, stellt eine „Herner“ Spitze dar. Das Stück unterscheidet sich nur durch die glatte Schlagfläche und die weiter auf die Fläche greifende Retuschierung von dem zuerst beschriebenen Beispiel. Aus Herne liegt weiter ein kleiner Schildkern vor.

Ganz entsprechend ist die Zusammensetzung der von K. H. JaCOB-Friesen (1949) bekanntgegebenen Leinetalfunde. Die Faustkeilformen von $\mathrm{Hannover-Döhren} \mathrm{:}$ I.angschmale, spitze Stücke mit gleichmäßig verdicktem Ende (JACOB-FrIESEN 1949, Abb. 8 - S. 33); breitdreieckige Faustkeile, nur unvollständig durchgearbeitet (ebda, Abb. 9 S. 35); massive breitdreieckige Faustkeile, über $10 \mathrm{~cm}$ lang (z. B. ebda, Abb. 5/6 - S. 27-29). Unter den massiv-breitdreieckigen Stücken gesondert $\mathrm{zu}$ nennen ist ein schweres, regelmäßiges Werkzeug (ebda, Abb. 1/2 - S. 19-21). Wie in Lebenstedt und Herne, kommen auch in Döhren blattförmige Schaber mit bogenförmiger, dorsal und ventral geschärfter Arbeitskante (ebda, Abb. 20 - S. 57) vor. Die der Arbeitskante gegenüberliegende Kante ist stets gröber, oft in sich alternierend behauen. Gut vertreten sind Schmalklingen (ebda, Abb. 17/18 - S. 51-53), dazu zahlreiche Levalloisabschläge (ebda, Abb. 14/15 - S. 45-47) und einige Clactonabschläge (in Hannover, Niedersächsisches Landesmuseum, liegt noch eine größere Anzahl von ЈАСОВ-Friesen nicht abgebildeter Clacton- und Levalloisabschläge).

Wichtigste Kernsteinform sind auch in Döhren Schildkerne. Dazu kommt ein konischer Nukleus mit glatter Standfläche (Abb. 8, 1), der seine genaue Entsprechung in einem Lebenstedter Stück findet.

Aus $\mathrm{R}$ e the n liegt mit einem langschmalen, spitzen Faustkeil (JACOB-FriesEN 1949, Abb. 25/26 - S. 67-69) eine aus den vorstehend beschriebenen Inventaren schon hinreichend bekannte Werkzeugform vor. Eine Besonderheit ist dagegen der große, langgestrecktovaleFaustkeil (ebda, Abb. 21/22 - S. 59-61), der einer limande nahekommt. Durch das verdickte, stumpfe Ende kann das Stück auch in die Nähe der oben genannten langschmalen Faustkeile gestellt werden. Annähernd dreieckige Faustkeile sind in Rethen zahlreich. Auch hier sind diese Werkzeuge in der Regel nicht völlig durchgearbeitet (ebda, Abb. 30 S. 77). Massive breitdreieckige Faustkeile fehlen nicht (ebda, Abb. 23/24 - S. 63-65).

Von den blattförmigen Schabern (ebda, Abb. 47 - S. 111) war schon wiederholt die Rede. Für die Publikationserlaubnis der beiden schönen Stücke Abb. 6, 1 und Abb. 7, 1 danke ich Herrn W. AdRIAN, Bielefeld. Erst nach der Veröffentlichung durch K. H. JACOBFrIESEN gefunden wurde ein kleinerer blattförmiger Schaber, der hiermit nachgetragen sei (Abb. 7, 2). Ferner sind einfache Schaber mit leicht konvexer, nur dorsal retuschierter Arbeitskante parallel zur Schlagrichtung vorhanden (Abb. 6, 2). Wie an den übrigen Fund-

3) Zeichnungen: H. Bosinski und E. Prokop. 


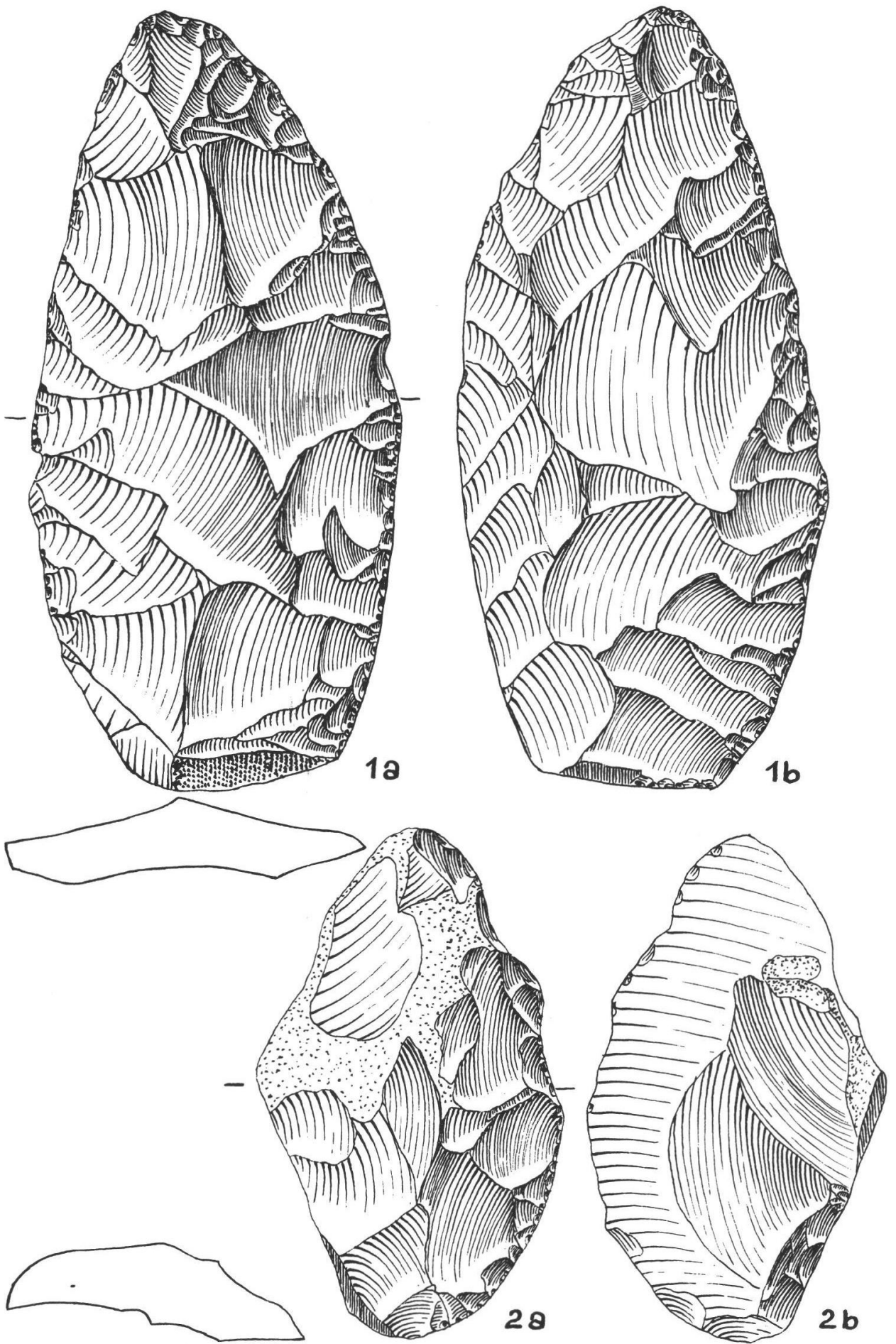

Abb. 6. Rethen (1:1). Blattförmiger Schaber (1) und einfacher Schaber (2). 

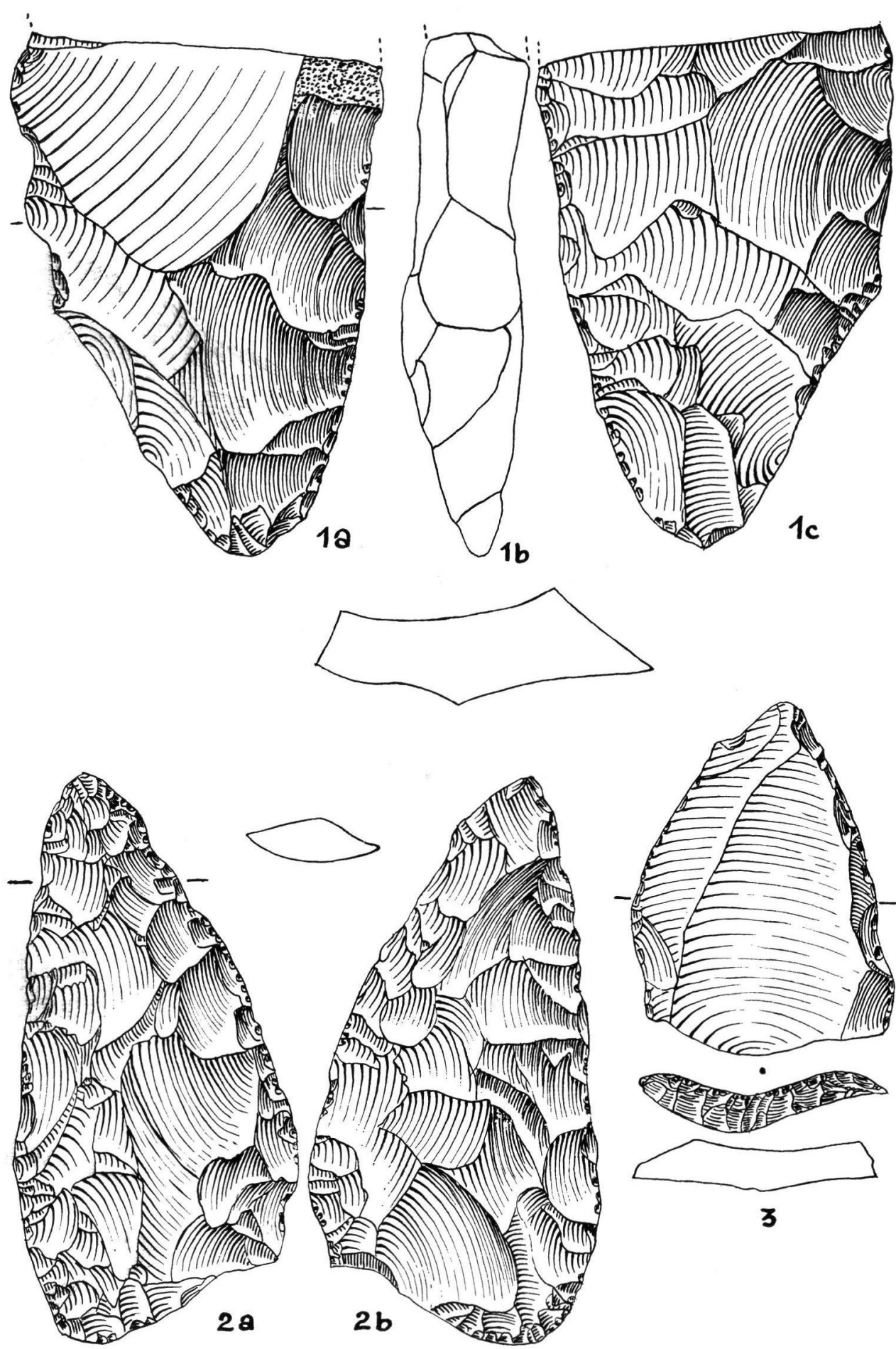

Abb. 7. Rethen (1:1). Blattförmige Schaber (1-2) und Levallois-Spitze (3). 

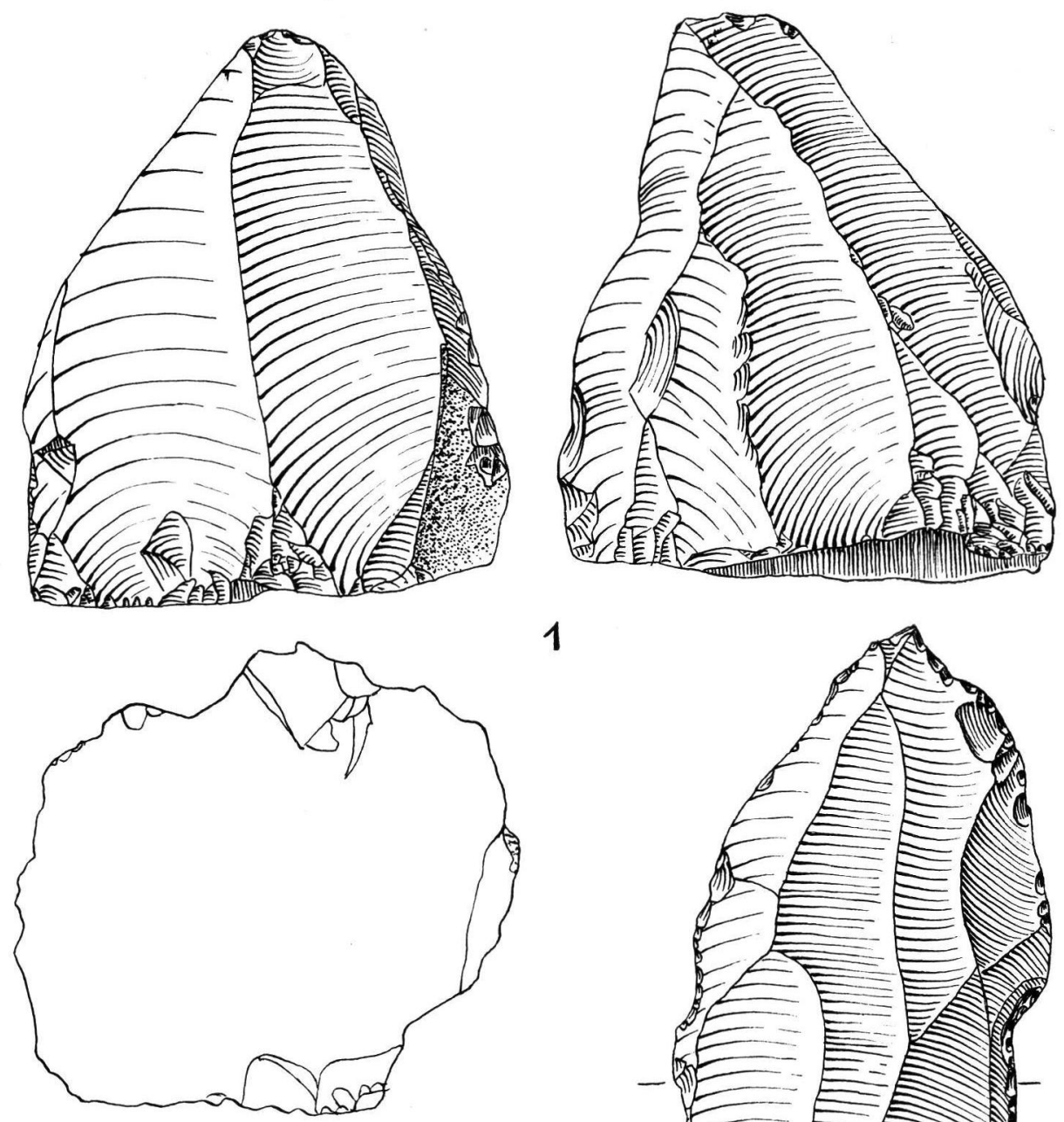

1

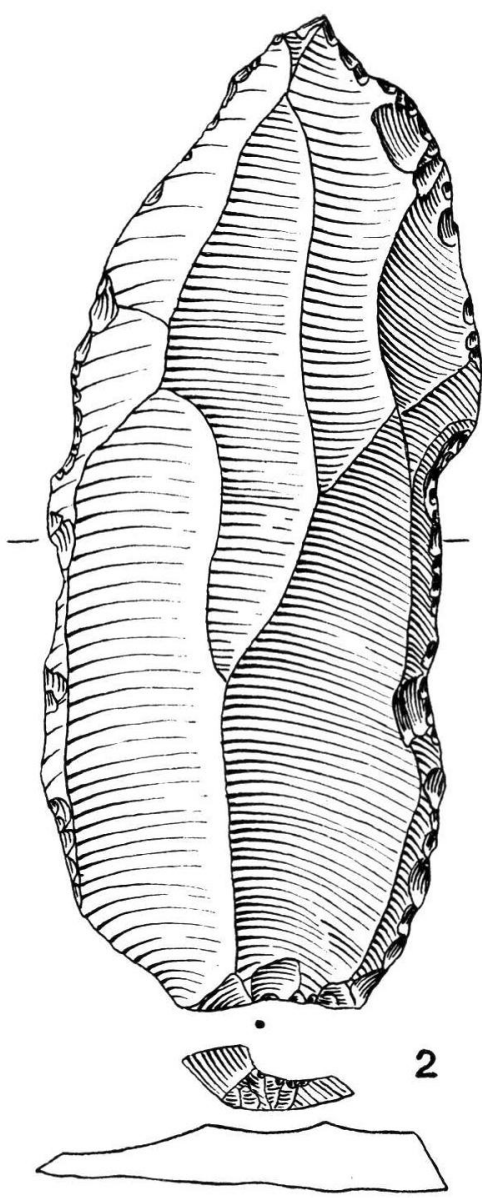

Abb. 8. Hannover-Döhren (1) und Rethen (2) (1:1). Konischer Kernstein (1) und Levallois-Abschlag (2). 
plätzen fehlen auch in Rethen Breitschaber, Doppelschaber, Winkelschaber und Spitzschaber. Einzige Spitzenform sind Levalloisspitzen (Abb. 7, 3). Klingen, an den Kanten mitunter ausgesplittert, kommen vor (JACOB-FrIESEN 1949, Abb. 46 - S. 109). Die Großklinge ebda, Abb. 44 - S. 105 stellt lediglich eine Sonderform der in Rethen ebenfalls zahlreichen breitflachen Levalloisabschläge (Abb. 8, 2) dar. Im Museum Hannover liegt auch ein Schildkern aus Rethen.

Wichtig für die relative Chronologie sind die wenigen Funde aus der untersten Fundschicht der Balver Höhle (Günther 1961), deren Studium vor der endgültigen Publikation ich K. Günther, Münster, verdanke. Vorhanden sind annähernd breitdreieckige, nicht völlig durchgearbeitete Faustkeile, blattförmige Schaber (in der Umrißgestaltung nicht ganz typisch), einfache Schaber mit einer nur dorsal retuschierten Arbeitskante parallel zur Schlagrichtung. Dazu einige Levalloisabschläge und Schildkerne, besonders erwähnenswert ein regelmäßiges, scheibenförmiges Stück.

Die Anführung weiterer Funde - kleinere Inventare und Einzelfunde - aus dem nordwestdeutschen Raum ist in diesem Zusammenhang unwesentlich. Ich möchte auf meine eingangs angeführte Arbeit verweisen.

\section{B. Formenkundliche Charakteristik}

Der Formenschatz der oben beschriebenen Inventare enthält (in der Reihenfolge des Typenspektrums Abb. 9) die nachstehenden Formen:

1. Langschmale, spitze Faustkeile mit kontinuierlich verdicktem Ende und in der Regel symmetrischer, auf der Mittelachse liegender Spitze.

2. Annähernd dreieckige, kleinere Faustkeile, meist nur unvollständig durchgearbeitet.

3. Massive, über $10 \mathrm{~cm}$ lange Faustkeile, oft nur an den Kanten und an der Spitze besser bearbeitet, dazu an den Längskanten häufig alternierend retuschiert, so daß der Querschnitt sich einem Parallelogramm nähert.

4. Blattförmige Schaber mit bogenförmiger, beidflächig retuschierter Arbeitskante.

5. Einfache Schaber mit einer konvexen oder geraden, nur dorsal retuschierten Arbeitskante parallel zur Schlagrichtung; die Schlagfläche ist häufig fazettiert. Andere Schaberformen, insbesondere Breitschaber (racloirs transversaux) und Schaber mit zwei Arbeitskanten (Doppelschaber, Winkelschaber, Spitzschaber) fehlen.

6. Levalloisspitzen, an den Kanten mitunter partiell retuschiert.

7. „Herner“ Spitzen.

8.-11. Regelmäßige Klingen gehören zum festen Bestand. Hin und wieder sind sie an einer oder beiden Längskanten dorsal retuschiert.

12.-13. Zahlreiche Abschläge von klassifikatorischer Bedeutung sind mitbestimmend für den Charakter der in Rede stehenden Formengruppe. Es kommen Clacton- und Levalloisabschläge vor, letztere stets in der Mehrzahl.

14. Schildkerne stellen die wichtigste Kernsteinform dar.

15. Konische Kernsteine liegen aus Lebenstedt und Hannover-Döhren vor.

16. Strunkförmige Nuklei (Nucleus Levallois à lames: Bondes 1961, Taf. 104) wurden gleichfalls hin und wieder angetroffen.

Im Interesse einer exakten, eindeutigen Erfassung wird die so charakterisierte Formengruppe vorerst als Lebenstedter Gruppe bezeichnet.

Innerhalb des mitgeteilten, unverwechselbaren Typenspektrums haben Leitformcharakter bzw. sind in anderen Verbänden sehr selten (in Abb. 9 durch Unterstreichung gekennzeichnet): Langschmale Faustkeile mit gleichmäßig verdicktem Ende, massive breit- 

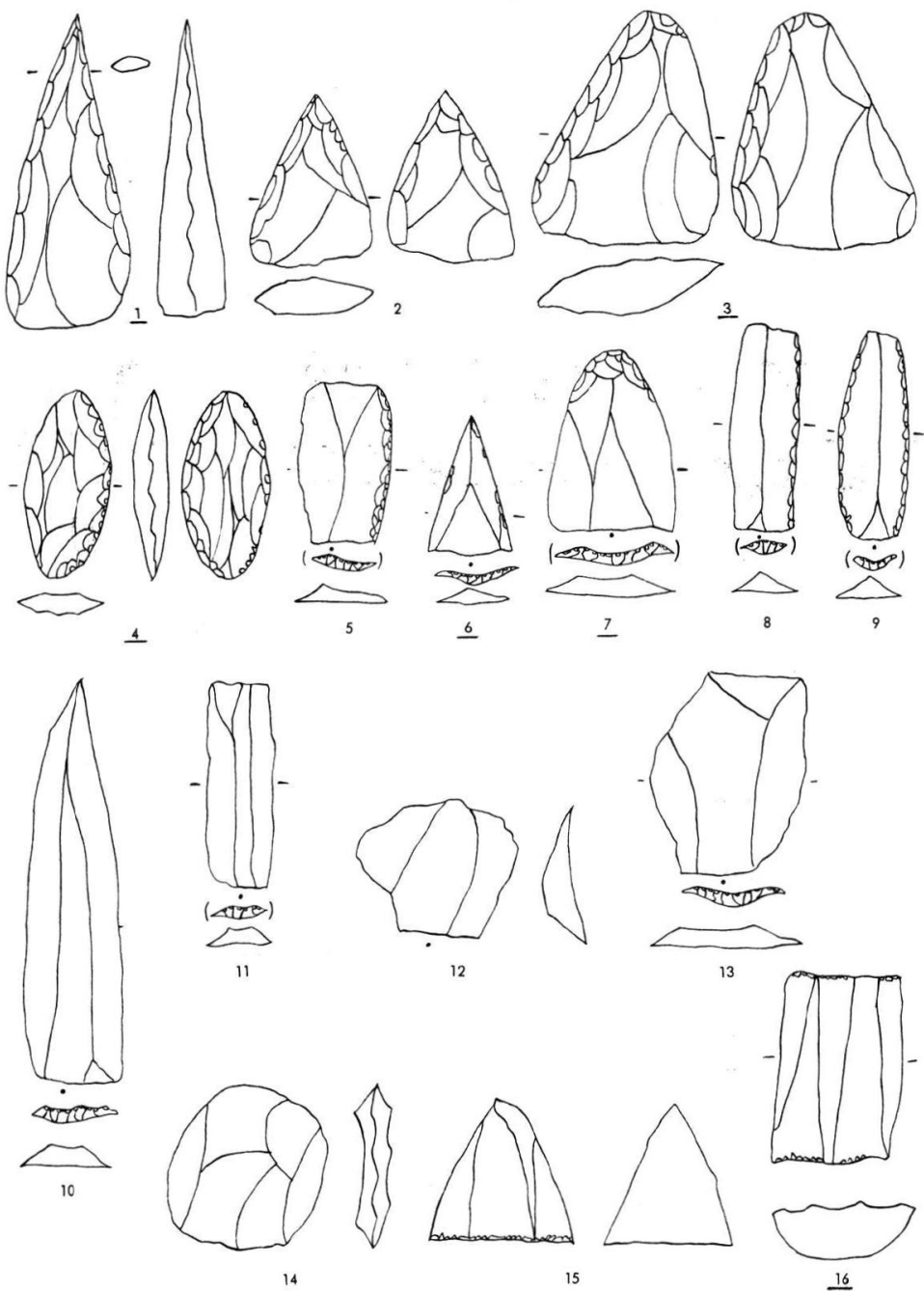

15

Abb. 9. Typenspektrum. Schema, ca. $1: 4$.

dreieckige Faustkeile, blattförmige Schaber, große Levalloisspitzen, „Herner“ Spitzen und strunkförmige Nuklei; in gewissem Umfang auch größere, typische Levalloisabschläge und gleichfalls größere, regelmäßige Schildkerne.

\section{Verbreitung nordwärts der europäischen Hochgebirgskette}

Funde der Lebenstedter Gruppe sind auch aus Mitteldeutschland bekanntgeworden. $\mathrm{Hund}$ is burg wurde neuerdings von V. Toepfer (1961) zusammenfassend veröffentlicht. Danach sind insgesamt 101 Artefakte vorhanden, deren Zusammensetzung völlig den bisher geschilderten Verbänden entspricht: Langschmaler, spitzer Faustkeil (TOEPFER 1961, Abb. 2, 1), blattförmiger Schaber (ebda, Abb. 3, 6 - als Faustkeil angesprochen), ein weiteres doppelflächig bearbeitetes Werkzeug (ebda, Abb. 2, 3). Dazu einfache Schaber (ebda, Abb. 2,5), Klingen, oft mit fazettierter Schlagfläche (ebda, Abb. 3, 8), zahlreiche breitflache Levalloisabschläge (ebda, Abb. 4 und Abb. 7). 
Hier anzuschließen sind die Funde von Markkle eberg (Grahmann 1955): Langschmale Faustkeile (ebda, Fig. 72, 4; Fig. 75, 1 mit abgebrochener Spitze; bei etwas anderer Ergänzung auch Fig. 74, 3); blattförmige Schaber (ebda, Fig. 72, 3+7; Fig. 73, 1; weitere blattförmige Schaber wurden von F. DAvis (1961) bekanntgegeben). Einfache Schaber sind gut vertreten (GrahmanN 1955, Fig. 65), darunter Stücke mit fazettierter Schlagfläche (ebda, Fig. 63, 3+8; Fig. 64, 8-10). Unter den Spitzen sind besonders große Levalloisspitzen mit geraden Kanten zu nennen (ebda, Fig. 53, 12; Fig. 56, 7). Gut repräsentiert sind Klingen mit einer (ebda, Fig. 48, 14) oder zwei (ebda, Fig. 47, 2+4+11-12) dorsal retuschierten Längskanten. Zahlreich sind Klingen ohne weitere Bearbeitung, häufig mit fazettierter Schlagfläche. Clactonabschläge mit glatter, großer Schlagfläche und stumpfem Schlagwinkel sind gut vertreten, Levalloisabschläge mit über 400 Beispielen belegt. Schildkerne kommen mit typischen Stücken vor. In Köln (Institut für Ur- und Frühgeschichte) liegt ferner ein großer, strunkförmiger Kernstein (Nucleus Levallois à lames).

Die Fundstelle $\mathrm{Zehmen} \mathrm{liegt} \mathrm{nur} \mathrm{wenige} \mathrm{Kilometer} \mathrm{südlich} \mathrm{von} \mathrm{Markkleeberg,}$ ebenfalls auf der Mittelterrasse der Pleisse. Das Inventar ist ganz entsprechend: Langschmaler Faustkeil (Grahmann 1955, Fig. 75, 2), Spitzenbruchstück eines weiteren Faustkeils (ebda, Fig. 72, 2). Levalloisspitzen (ebda, Fig. 53, 5), Klingen und das Bruchstück einer an beiden Längskanten dorsal retuschierten Klinge (ebda, Fig. 47, 3) sowie Clactonund Levalloisabschläge fügen sich ohne weiteres in das bisher gezeichnete Bild.

Als größerer Verband ist schließlich noch $\mathrm{Cr}$ öbe $\mathrm{rn}$, zwischen Markkleeberg und Zehmen gelegen, zu erwähnen. Die Zusammensetzung des Inventars ist die gleiche (GRAHManN 1955, S. 563), nur daß in Cröbern Faustkeile fehlen. Das Fehlen der Faustkeile in dem kleineren Verband (insgesamt ca. 100 Artefakte) ist nicht überraschend, denn zahlreich waren sie an keinem Platz der Lebenstedter Gruppe.

Einzelfunde der Lebenstedter Gruppe sind außer aus der Leipziger Gegend ( L e i p zig-W a hren: Grahmann 1955, Fig. 62, 6; Le i p z ig-Lin d e n a u : ebda, S. 518) auch aus Elbschottern (Gerw is ch: Toepfer 1959, 1959a; B a r leben : JAHN 1951) u.a. (z. B. Ma n n ha u s en = Et inge n : WIEgERS 1939/40) bekanntgeworden. Weiter östlich gibt es nur vereinzelte Vorkommen. Der massiv-breitdreieckige Faustkeil von K r e š i c e bei Leitmeritz (KERN 1932, MAZÁLEK 1953), die Funde von M a k a u (Lindner 1937, ChMielewski 1955) und K o r n i t z (Lindner 1937, KozŁowski 1961, S. 119) im Kreise Ratibor sowie der Schildkern von Oderf u r t (Lindner 1956) kennzeichnen den östlichsten Teil des zusammenhängenden Verbreitungsgebietes.

Nur erwähnt werden sollen vergleichbare Funde viel weiter im Osten, in Armenien. Als wichtiger Platz Dshraber in der Nähe von Jerewan (Ljubin 1961). Charakteristische Stücke dieses Inventares sind Faustkeile (ebda, Abb. 29), Levalloisspitzen (ebda, Abb. 31, 1), Levalloisabschläge (ebda, Abb. 30 und Abb. 31) und Schildkerne (ebda, Abb. $30,4)$. Eine Verbindung der armenischen Vorkommen mit den mitteleuropäischen Funden nordwärts der Gebirgskette (Alpen-Kaukasus) dürfte ausscheiden; jedenfalls sind aus dem großen, dazwischenliegenden Gebiet - vielleicht mit Ausnahme der Artefakte von L u k a Wrublewezka ja (hier beschränkt sich die Ahnlichkeit auf den Faustkeil BorisKowski 1953, Abb. 14 - S. 52, während Levalloisformen fehlen) - nur gänzlich andersartige Funde bekanntgeworden. Auf entsprechende Funde südlich der Gebirgskette kann hier nicht eingegangen werden.

Das Zentrum der Verbreitung liegt in Westeuropa. Besonders aus Nordfrankreich sind zahlreiche Fundstellen bekanntgeworden (vgl. etwa Bordes 1954). Die Funde werden dort als Acheuléen supérieur bezeichnet. Wichtig erscheint, daß die blattförmigen Schaber auch hier zum festen Formenschatz gehören (KELLEY 1960). Im südlichen England sind derartige Komplexe ebenfalls gut belegt. So setzt sich das "Levalloisien“ von B a ke r s Hole (SMith 1911, OAKLey \& King 1945, Breuil 1947) in den wichtigsten Formen fol- 
gendermaßen zusammen (Funde im British Museum, London): Langschmale Faustkeile niit kontinuierlich verdicktem Ende (9 Stücke), kleinere, annähernd dreieckige, nicht völlig durchgearbeitete Faustkeile (5), einfache Schaber, z. T. an typischen Levalloisabschlägen (5), 1 große Levalloisspitze, regelmäßige Klingen (14), Großklingen (2), breitflache, größere Levalloisabschläge (103), meist größere Schildkerne (15), strunkförmige, teilweise sehr massive Kernsteine (6).

Schließlich sind noch manchmal bestrittene Vorkommen des Acheuléen supérieur (nicht Micoquien im Sinne des aus der obersten Fundschicht von L a M i c o qu e vorliegenden Verbandes) aus Südfrankreich zu erwähnen: Im Institut de Paleontologie Humaine, Paris, liegen zweifelsfrei hier anzuschließende Funde aus dem Abri "Eg lis e de Guilh e m " bei Les Eyzies (Dordogne) (ohne Abbildungen publiziert: Capitan, Breuil \& Peyrony 1902), u. a. langschmaler Faustkeil, massiv-breitdreieckiger Faustkeil, einfache Schaber, Klingen, Levalloisabschläge, Schildkern. Die Faustkeile sind zu groß, um im „Moustérien de tradition acbeuléenne" vorzukommen, abgesehen davon, daß sämtliche Leitformen dieser Kultur wie rückengestumpfte Messer und doppelflächig retuschierte Spitzen fehlen.

\section{Geochronologie}

Die hinsichtlich der geochronologischen Einordnung bestehende Problematik wird an einigen deutschen Funden aufgezeigt. Durch eine Uberlagerung in der Balver Höhle ist die Lebenstedter Gruppe in diesem Fall als älter als das Micoquien ausgewiesen. Die Fauna aller Fundstellen ist, soweit entsprechende Untersuchungen vorliegen, einheitlich: Ren, Mammut, Wisent, Wildpferd, Wollnashorn, in der Balver Höhe lediglich Wollnashorn und Höhlenbär. Das Gleiche gilt für die Flora. Sie besteht zum überwiegenden Teil aus Gräsern (Gramineen und Cyperaceen, Polargrasnelke, Kuckucksnelke); daneben kommen Polarweide, kriechende Weide, Zwergbirke, vereinzelt Kiefer und Fichte vor (Tab. 1).

Tabelle 1

Fauna, Flora und Lagerungsverhältnisse an wichtigen Fundplätzen der Lebenstedter Gruppe.

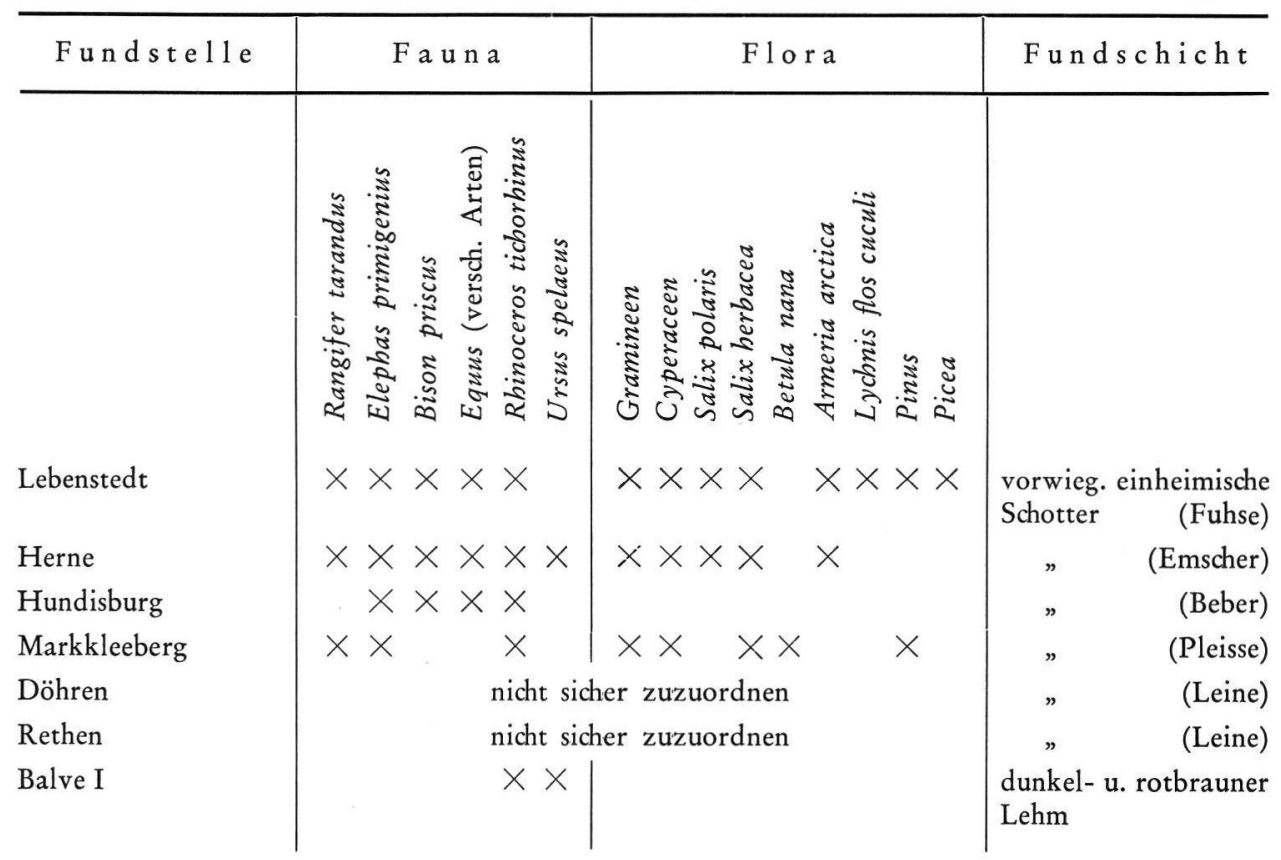


Als großräumiges Biotop wurde eine grasreiche Tundra mit einzelnen Bäumen und Sträuchern angenommen (für Lebenstedt: KLeinschmidt 1953, Selle 1953, Pfaffenberg 1953). Das einbettende Sediment der Fundschichten sind mit Ausnahme der Balver Höhle stets in ihrer Zusammensetzung vorwiegend einheimische Flußschotter.

Fauna und Flora datieren die Funde der Lebenstedter Gruppe übereinstimmend in eine Kaltphase (vielleicht mit Ausnahme der untersten Schicht der Balver Höhe, da hier entsprechende Angaben fehlen). Die Funde von Hundisburg lagen unter einer Grundmoräne. Die Fundschicht wurde von V. Toepfer (1961, S. 36/37) in den Beginn der Saaleeiszeit datiert; die Argumentation, daß die Artefakte in der Fundschicht sekundär seien, weil die Menschen nur am Ende eines Interglazials ausreichende Lebensbedingungen vorfanden (ebda, S. 38), ist durch andere, sicher kaltzeitliche Funde widerlegt.

Die Funde von Markkleeberg, Zehmen und Cröbern werden ebenfalls in die erste Hälfte der Saaleeiszeit datiert (Grahmann 1955, S. 523; ein formenkundlich unbedeutender und hier nicht interessierender Teil der Funde ist abgerollt und stark patiniert und könnte nach GrahmanN in das vorausgegangene Interglazial gehören.)

Die Fundbergung durch einen auf dem Baggersee schwimmenden Bagger ließ in Hannover-Döhren und Rethen keine genaueren Profilbeobachtungen zu. Aufgrund des auf der Mittelterrasse der Leine gelegenen Profils von A r n u m (НАмм 1949, S. 120), dem ebenfalls unter einer Grundmoräne ein Levalloisabschlag entnommen wurde, der zur Lebenstedter Gruppe gehören dürfte (JACOB-Friesen 1949, Abb. 49/50 - S. 115-117), werden jedoch auch Rethen und Hannover-Döhren meist in die Saaleeiszeit datiert.

Die Ansetzung der Herner Funde ist unterschiedlich. E. KaHRs (1925) trat für eine Datierung in die Saaleeiszeit ein, andere Forscher stellten die Funde an den Beginn der Würmeiszeit (z. B. Andree 1939, S. 35) Lebenstedt selbst schließlich wurde von F. Preul. (1953) in die Würmeiszeit datiert. PREUL schließt ein saaleeiszeitliches Alter nicht vollständig aus (ebda, S. 154).

C14-Untersuchungen ergaben für Lebenstedt $48300 \pm 2000$ Jahre (DE Vries 1958, S. 14) und $55000 \pm 1000$ Jahre (Gro 2083), also Werte, die in das frühe Würm fallen.

\section{E. Auswertung}

Die Tatsache, daß es ein Levalloisien als eigene Kultur zumindest in Europa nicht gibt, ist nicht neu (z. B. Bordes 1950). Die Theorie vom Gegensatz der Faustkeil- und Abschlagkulturen (BreuIL 1932) hat sich ihrer leichten Faßlichkeit wegen jedoch weit ausgebreitet. Die Unterscheidung von Levalloisien und Acheuléen wird durch Funde nicht gestützt. Es handelt sich vielmehr um einen einheitlichen Komplex, dessen eine Komponente Faustkeile sind und dessen zweite Komponente die Levalloistechnik ist. In ähnlicher Weise wurde das Acheuléen schon 1889 durch G. D'Ault Du Mesnil (1889) definiert.

Ein derartiger, dem Acheuléen anzuschließender Komplex liegt mit der L ebenst edter Grup pe vor. Die Lebenstedter Gruppe besitzt einen einheitlichen Formenschatz und eine geschlossene Verbreitung. Darüber hinaus ist das Klima einheitlich kalt. Diese Tatsachen sprechen für eine einheitliche zeitliche Ansetzung.

Nach bisher vorliegenden geologischen Untersuchungen ist dies jedoch nicht der Fall. Die meisten Funde sind in die Saale-Eiszeit datiert worden, während in Salzgitter-Lebenstedt ein würmzeitliches Alter angenommen wurde. Da die saaleeiszeitliche Datierung u. a. von Hundisburg und Markkleeberg unbestritten $\mathrm{zu}$ sein scheint, ergeben sich bei einer Datierung von Salzgitter-Lebenstedt ins Würm aus urgeschichtlicher Sicht folgende Schwierigkeiten:

1. Ein Nachleben dieser Kultur über das ganze Eem, im Formenschatz völlig unverändert, erscheint umso weniger glaubhaft, als aus dem Eem keine Fundstellen bekannt 
sind, die zwischen den saaleeiszeitlichen und den würmeiszeitlichen Vorkommen der Lebenstedter Gruppe vermitteln könnten. So hat z. B. die Micoquien am Ende des Eems mit der Lebenstedter Gruppe nicht das Mindeste zu tun. Hier muß auch die Überlagerung der Lebenstedter Gruppe durch Micoquien in der Bxlver Höhle nochmals erwähnt werden.

2. Die aus dem frühen Würm bekannten mitteloaläolithischen Kulturen, insbesondere im weitesten Sinne als Moustérien zu bezeichnende Verbände, sind in ihrem Formenschatz scharf von der Lebenstedter Gruppe unterschieden und zeigen auch keinerlei Beeinflussungen (und umgekehrt). Lediglich allgemein-mittelpaläolithische Formen wie einfache Schaber sind überall vorhanden.

So besteht das Anliegen dieser Darstellung darin, die aus urgeschichtlicher Sicht einheitlichen Stationen der Lebenstedter Gruppe der Geologie zur erneuten chronologischen Überprüfung zu empfehlen. Die Frage ist, konkret ausgedrückt, also: Kann SalzgitterLebenstedt geologisch auch älter sein oder können die übrigen Funde der Lebenstedter Gruppe auch dem Frühwürm angehören?

\section{Literaturverzeichnis}

Andree, J.: Der eiszeitliche Mensch in Deutschland und seine Kulturen. - Stuttgart 1939.

Ault du Mesnil, G. DE: Étude des deux versants de la vallée de la Somme à Abbeville. - La Société, l'École et le Laboratoire d'Anthropologie de Paris à l'Exposition universelle de 1889, S. 145 ff. Paris 1889.

Bordes, F.: L'évolution buissonnante des industries en Europe occidentale. Considérations théoriques sur le paléolithique ancien et moyen. - L'Anthropologie 54, S. $393 \mathrm{ff}$., Paris 1950. - Les limons quaternaires du bassin de la Seine. - Archives de l'Institut de Paléontologie humaine, Memoire 26, Paris 1954. - - Typologie du paléolithique ancien et moyen Bordeaux 1961

Boriskowsкi, P. I.: Paleolit Ukrainy, - Materialy i issledowanija po archeologii SSSR 40, Moskau und Leningrad 1953.

Breuil, H.: Les Industries à eclats du Paléolithique Ancien. I. Le Clactonien. - Préhistoire 1, S. $125 \mathrm{ff}$., Paris 1932. - - Age of the Bakers Hole Coombe Rock, Northfleet, Kent. Nature 160, S. $831 \mathrm{ff} .$, London 1947.

Capitan, L., Breuil, H. ex Peyrony, D.: La station acheuléenne de la grotte-abri "L'Eglise de Guilhem" près des Eyzies (Dordogne). - Association Franc. pour l'Avancement des Sciences, S. 769 ff., 1902.

Chmielewski, M. und W.: Stanowisko paleolityczne w Makowie (Etude du gisement paléolithique du Maków). - Biuletyn Peryglacjalny 2, S. 58 ff., Łodz 1955.

Davis, F. D.: Blattformen von Markkleeberg. - Quartär 13, S. 93 ff., Bonn 1961.

Grahmann, R.: The Lower Palaeolithic Site of Markkleeberg and other comparable Localities near Leipzig. - Trans. Amer. Phil. Soc., N. S. 45, S. 509 ff., Philadelphia 1955.

GüNTHER, K.: Vorgeschichtliche Funde aus den westfälischen Höhlen. - Jahreshefte für Karst- und Höhlenkunde, S. $261 \mathrm{ff}$., München 1961.

Hamm, F.: Über die eiszeitlichen Ablagerungen im Leinetal südlich von Hannover als Fundpunkte von Altpaläolithen. - in: JАСOB-FrIESEN 1949, S. $119 \mathrm{ff}$.

JaCob-Friesen, K. H.: Die Altsteinzeitfunde aus dem Leinetal bei Hannover. - Hildesheim 1949.

JAHN, M.: Ein neues Altsteinzeitgerät aus Barleben. - Jahresschrift für mitteldeutsche Vorgeschichte 35, S. 5 ff. - Halle 1951.

F.AHrs, E.: Pa'äolithische Funde aus dem Diluvium des Emschertales. - Prähistorische Zeitschrift 16, S. $93 \mathrm{ff}$., Berlin 1925. - - Das Diluvium des Emscher-Gebietes und seine paläolithischen Kulturreste. - Tag.-Ber. der Anthrop. Ges. in Köln, Leipzig 1928.

Kelley, H.: Bifaces acheuléens de forme foliacée. - Bull. de la Société préhistorique franc. 57, S. $480 \mathrm{ffi}$., Le Mans 1960.

Kern, J.: Der Faustkeil von Krscheschitz a. E. - Sudeta 8, S. 75 ff., - Bodenbach 1932.

Kleinschmidt, A.: Die zoologischen Funde - in: Tode 1953, S. $166 \mathrm{ff}$.

KozŁowski, J.: Bemerkungen über den Stand der Paläolithforschung in Polen. - Archaeologia Austriaca 30, S. 118 ff., 1961.

LinDNER, H.: Die Eiszeiten und der eiszeitliche Mensch im südlichen Oberschlesien. - Jahresb. d. Geologischen Vereinigung Oberschlesiens 1937, S. 1 ff., Gleiwitz 1937. - - Die geologische Datierung des Schildkerns von Oderfurt. - Quartär 7/8, S. 188 ff., Bonn 1956. 
LJubiN, W. P.: Verchneašel'skaja apojanka Dshraber (Armenija). - Kratkie Soobščenija o dokladach i polewych issledowanijach instituta archeologii 82, S. 59 ff., Moskau 1961.

MazÁLeK, M.: Přrispěvek k historii výzkumu starého paleolitu Čech (Beitrag zur Geschichte der Durchforschung des Altpaläolithikums Böhmens). - Anthropozoikum 3, S. $191 \mathrm{ff}$., Prag 1953.

Oaklex, K. P. and KIng, W. B. R.: Age of the Bakers Hole Coombe Rock, Northfleet, Kent. Nature 155, S. 51 f., London 1945.

Pfaffenberg, K.: Pflanzenreste aus den Fundschichten von Lebenstedt. - in: Tode 1953, S. $163 \mathrm{ff}$.

Preul, F.: Die geologische Bearbeitung. - in: Tode 1953, S. $149 \mathrm{ff}$.

SELLE, W.: Pollenanalythische Untersuchungen. - in: Tode 1953, S. $161 \mathrm{ff}$.

Smith, R. A.: A Palaeolithic Industry at Northfleet, Kent. - Archaeologia 62, S. 515 ff., 1911.

TODE, A.: Die Untersuchung der paläolithischen Freilandstation von Salzgitter-Lebenstedt. 8. Einige archäologische Erkenntnisse. - Eiszeitalter und Gegenwart 3, S. 192 ff., 1953.

Toepfer, V.: Der Faustkeil von Gerwisch bei Magdeburg. - Ausgrabungen und Funde 4, S. $5 \mathrm{ff}$., Berlin 1959. - - Ein Faustkeil aus der Umgebung von Magdeburg. - Jahresschrift für mitteldeutsche Vorgeschichte 43, S. 22 ff., Halle 1959(a). - - Die altpaläolithischen Feuersteinwerkzeuge von Hundisburg. - Jahresschrift für mitteldeutsche Vorgeschichte 45, S. 35 ff. - Halle 1961.

DE VRIEs, HI.: Radiocarbon dates for upper Eem and Würminterstadial samples. - Eiszeitalter und Gegenwart 9, S. 10 ff. - 1958.

Wiegers, F.: Ein Acheul-Faustkeil von Etingen (Altmark). - Prähistorische Zeitschrift 30/31, S. 35 ff., Berlin 1939/40.

Manuskr. eingeg. 18. 12. 1962.

Anschrift des Verf.: Gerhard Bosinski, 5 Köln, Weyertal 125, Institut für Ur- und Frühgeschichte. 